\title{
SIMULATION AND IDENTIFICATION OF HYDRONIC RADIATOR MODEL AS A FIRST STEP TOWARDS EFFICIENT CONTROL OF THERMAL ENERGY CONSUMPTION IN ZONES
}

\author{
Ivan Bevanda, Petar Marić
}
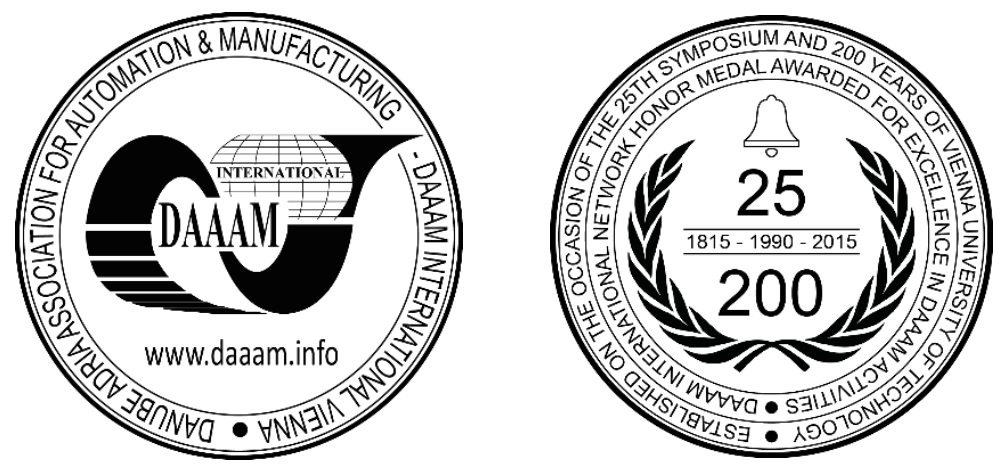

This Publication has to be referred as: Bevanda, I[van] \& Maric, P[etar] (2018). Simulation and Identification of Hydronic Radiator Model as a First Step Towards Efficient Control of Thermal Energy Consumption in Zones, Proceedings of the 29th DAAAM International Symposium, pp.1076-1083, B. Katalinic (Ed.), Published by DAAAM International, ISBN 978-3-902734-20-4, ISSN 1726-9679, Vienna, Austria

DOI: $10.2507 / 29$ th.daaam.proceedings. 154

\begin{abstract}
The buildings sector is the largest energy consumer with over one-third of final energy consumption globally and HVAC systems are its main energy-consuming component. Energy efficient control of heating and cooling processes can give a great contribution to overall energy savings. In this paper a hydronic radiator model has been developed and simulated using Mathwork's dynamic simulation package Simulink. The mathematical model has been described using first order non-linear differential equations. The identification of unknown parameters has been carried out in Matlab using nonlinear programming solver. This procedure is the first step towards the model predictive control of the radiator since the estimated parameters are needed for calculation of maximum energy transmitted to the zone.
\end{abstract}

Keywords: energy management; hydronic radiator; system identification; nonlinear optimization

\section{Introduction}

Most people currently spend $90 \%$ of their daily lives indoors and relying on mechanical heating and air conditioning, thus leading to buildings becoming the largest energy consumers worldwide. The ratio of building energy consumption to total energy consumption increased from 33.7 \% to $41.1 \%$ between 1980 and 2010 in the U.S. [1]. Usually, controllers in Building Energy Management systems have comfort as the single objective (commonly indicated as the set-point temperature); reducing energy consumption is less addressed in the design of temperature controllers. A promising solution for BEMS is Model Predictive Control (MPC). MPC has several features that make it suitable for the problems encountered in intermittently heated buildings. First, it is able to use the occupancy schedule and weather forecasts for optimal temperature control. Second, MPC optimizes not only the comfort but also an energy criterion. As heating systems generally consume energy to provide thermal comfort, MPC makes a trade-off between energy savings and thermal comfort. Theoretically, MPC gives the best results as compared to other control algorithms [2]. One of the projects dealing with MPC in buildings is 3Smart (Smart Building - Smart Grid - Smart City).The project is co-funded by the European Union through Interreg Danube Transnational Programme, and partners from the following countries are involved: Croatia, Slovenia, Austria, Hungary, Bosnia and Herzegovina and Serbia. 
The main objective of the 3Smart project is to provide a technological and legislative setup for cross-spanning energy management of buildings, grids and major city infrastructures in the Danube Region. It will provide optimal economical value to energy-efficiency and renewable energy investment in the building and at the same time it will result in optimized costs on the grid side whereas grid and buildings will also interact through exchanging energy and prices data [3]. The first step in the project is to analyse the overall energy consumption in building which has been done by the group of authors [4] and then to proceed to the zone level. This paper focuses on one part of research within 3Smart project regarding energy management control in zones with hydronic radiators as its thermal actuators. A hydronic radiator is a type of heat exchanger designed to transfer heat from one medium to another for the purpose of heating or cooling. Regardless of its purpose (heating the environment or cooling the fluid supplied to it), it is always a source of heat to its environment. They are still most commonly used in central heating systems for heat distribution to each zone of the building, but devices like FCUs (Fan coil units) are taking their place. Detailed analysis of radiator thermodynamic behaviour is given with developed procedure for identification of a radiator tested on simulations. A mathematical model of a hydronic radiator has been described with first order non-linear differential equations following the principle that model has to be as much detailed as possible to be reliable representation of a real system, but at the same time simple enough to be easy to control. Several authors have made a detailed mathematical model of hydronic radiator with all nonlinearities included [5], [6], [7], but those models are too complex for control purposes. Then, a model consisting of one zone heated with hydronic radiator has been developed using Matlab/Simulink and the simulation has been performed. The values of system inputs (water inlet temperature, medium mass flow and zone air temperature) have been changed during the simulation to get wide range of data of radiator behaviour. The identification procedure has been carried out in Matlab to obtain the model parameters. This procedure is the first step towards the MPC of the radiators.

\section{Mathematical model}

A model is a representation of reality that retains its salient features. The first task is to identify the system under study. Modelling usually implies approximating the real geometry to an ideal geometry (assuming perfect planar, cylindrical or spherical surfaces, or a set of points, a given interpolation function, and its domain), approximating material properties (constant values, isotropic values, reference material values, extrapolated values), and approximating the heat transfer equations (neglecting some contributions, linearizing some terms, assuming a continuum media, assuming a discretization, etc.). Modelling material properties introduces uncertainties because density, thermal conductivity, thermal capacity, emissivity, etc., depend on the base materials, their impurity contents, bulk and surface treatments applied, actual temperatures, the effects of aging, etc. In most cases materials properties are modelled as uniform in space and constant in time for each material, but the worthiness of this model and the right selection of the constant-property values, requires insight [8].

There are three mechanisms of heat transfer in radiators that need to be described with mathematical equations:

- Forced heat convection from fluid (water in the tube) to the inner wall of the tube

- Heat conduction through the tube wall

- Coupled radiation and natural convection from the outer tube wall to the outside fluid (air)

The following assumptions have been made:

- Since the water inlet temperature $T_{\mathrm{w}}^{\text {in }}$ and water outlet temperature $T_{\mathrm{w}}^{\text {out }}$ differ significantly, the arithmetic average of those two temperatures was taken as the water temperature inside the radiator

$T_{\mathrm{w}}^{\mathrm{av}}=\frac{T_{\mathrm{w}}^{\mathrm{in}}+T_{\mathrm{w}}^{\mathrm{out}}}{2}$

- $\quad$ System is hydraulically balanced

The heat transfer processes (Fig. 1) are described by the following differential equation:

$m_{w} c_{w} \frac{d T_{\mathrm{w}}^{\mathrm{out}}}{d t}=q_{\mathrm{w}} c_{w}\left(T_{\mathrm{w}}^{\mathrm{in}}-T_{\mathrm{w}}^{\mathrm{out}}\right)-U_{0}\left(T_{\mathrm{w}}^{\mathrm{av}}-T_{\mathrm{z}}\right)^{n}$

or:

$\frac{d T_{\mathrm{w}}^{\text {out }}}{d t}=\frac{q_{\mathrm{w}}}{m_{w}}\left(T_{\mathrm{w}}^{\text {in }}-T_{\mathrm{w}}^{\text {out }}\right)-\frac{U_{0}}{m_{w} c_{w}}\left(T_{\mathrm{w}}^{\mathrm{av}}-T_{\mathrm{z}}\right)^{n}$ 
where $m_{w}$ is the mass of water inside the radiator, $c_{w}$ is the specific heat capacity of the water, $q_{w}$ is the medium mass flow of the water through the radiator and $T_{z}$ is the zone temperature. $P_{w}=q_{\mathrm{w}} c_{w}\left(T_{\mathrm{w}}^{\text {in }}-T_{\mathrm{w}}^{\text {out }}\right)$ represents the thermal power on the water side. $P_{\mathrm{t}, \mathrm{r}}=U_{0}\left(T_{\mathrm{w}}^{\mathrm{av}}-T_{\mathrm{z}}\right)^{n}$ represents the thermal power of a radiator affecting the zone where $U_{0}$ is the overall heat transfer coefficient and $n$ is the radiator exponent describing the type of the radiator. For standard hydronic radiators the value of $n$ is around 1.33. This is a dynamic model of a water radiator in the case when $q_{w} \neq 0$. In the identification process the unknown parameters will be $a=\frac{1}{m_{w}}, b=\frac{U_{0}}{m_{w} c_{w}}$ and $n$, so the equation (3) can be rewritten in the following form:

$\frac{d T_{\mathrm{w}}^{\mathrm{out}}}{d t}=a q_{w}\left(T_{\mathrm{w}}^{\mathrm{in}}-T_{\mathrm{w}}^{\text {out }}\right)-b\left(T_{\mathrm{w}}^{\mathrm{av}}-T_{\mathrm{z}}\right)^{n}$

Through the process of identification the values of $a, b$ and $n$ will be estimated, but there is still unknown parameter $U_{0}$ that needs to be identified because the transmitted thermal power between the water side and the air in the room needs to be known for the energy input control. It will be identified using the steady state values of transmitted thermal power $P_{\mathrm{t}, \mathrm{r}}$. The whole identification process is described in the section 4 .

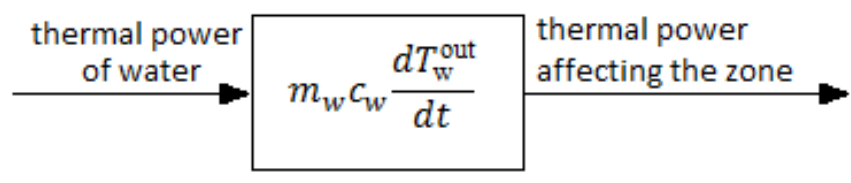

Fig. 1. Heat transfer processes of a radiator

It is also important to take into account a situation when there is no medium mass flow $\left(q_{w}=0\right)$. Values of medium mass flow and inlet water temperature are inserted in the equations (3) and (4) $\left(q_{w}=0\right.$ and $\left.T_{\mathrm{w}}^{\text {in }}=0\right)$ and the dynamic model for this case is described with equations (5) and (6):

$\frac{d T_{\mathrm{w}}^{\text {out }}}{d t}=-\frac{U_{0}}{m_{w} c_{w}}\left(T_{\mathrm{w}}^{\text {out }}-T_{\mathrm{z}}\right)^{n}$,

or

$\frac{d T_{\mathrm{w}}^{\text {out }}}{d t}=-b\left(T_{\mathrm{w}}^{\text {out }}-T_{\mathrm{z}}\right)^{n}$

\section{Simulation}

The detailed model consisting of a single zone and a hydronic radiator was made and simulated in Matlab/Simulink [9]. Simulink is a block diagram environment for multidomain simulation and model-based design. It supports systemlevel design, simulation, automatic code generation, and continuous test and verification of embedded systems. Simulink provides a graphical editor, customizable block libraries, and solvers for modelling and simulating dynamic systems. It is integrated with MATLAB, enabling incorporation of MATLAB algorithms into models and exportation of simulation results to MATLAB for further analysis [10]. The heat transfer processes in the radiator and between the radiator and the environment have been modelled by using the following equations:

$m_{w} c_{w} \frac{d T_{\mathrm{w}}^{\mathrm{out}}}{d t}=q_{\mathrm{w}} c_{w}\left(T_{\mathrm{w}}^{\mathrm{in}}-T_{\mathrm{w}}^{\mathrm{out}}\right)-U_{\mathrm{w}}\left(T_{\mathrm{w}}^{\mathrm{av}}-T_{\mathrm{r}}\right)$

$m_{r} c_{r} \frac{d T_{r}}{d t}=U_{\mathrm{w}}\left(T_{\mathrm{w}}^{\mathrm{av}}-T_{\mathrm{r}}\right)-U_{a}\left(T_{r}-T_{z}\right)^{n}$

where $m_{w}$ is the mass of the water inside the radiator, $c_{w}$ is the specific heat capacity of the water, $m_{r}$ is the mass of the radiator skin, $c_{r}$ is the specific heat capacity of the radiator, $q_{w}$ is the medium mass flow of the water through the radiator, $m_{a}$ is the mass of the air in the room, $c_{a}$ is the specific heat capacity of the water, $T_{r}$ is the radiator skin surface temperature, $T_{z}$ is the room temperature, $U_{w}$ is the heat transfer coefficient from the water to the radiator and $U_{a}$ is the heat transfer coefficient from the radiator skin surface to the air. This detailed mathematical model of a radiator has been taken as a representation of a real radiator and was simulated in Simulink. 
System inputs are inlet water temperature $T_{\mathrm{w}}^{\text {in }}$, medium mass flow $q_{w}$ and room temperature $T_{z}$. System outputs are outgoing radiator temperature $T_{\mathrm{w}}^{\text {out }}$ and radiator skin surface temperature $T_{r}$. For the purpose of the identification, the inlet water temperature and medium mass flow (Fig. 2. and Fig 3.) have been changed over time during the simulation to obtain the reliable data of system behaviour with different values so the system parameters could be found more accurately.

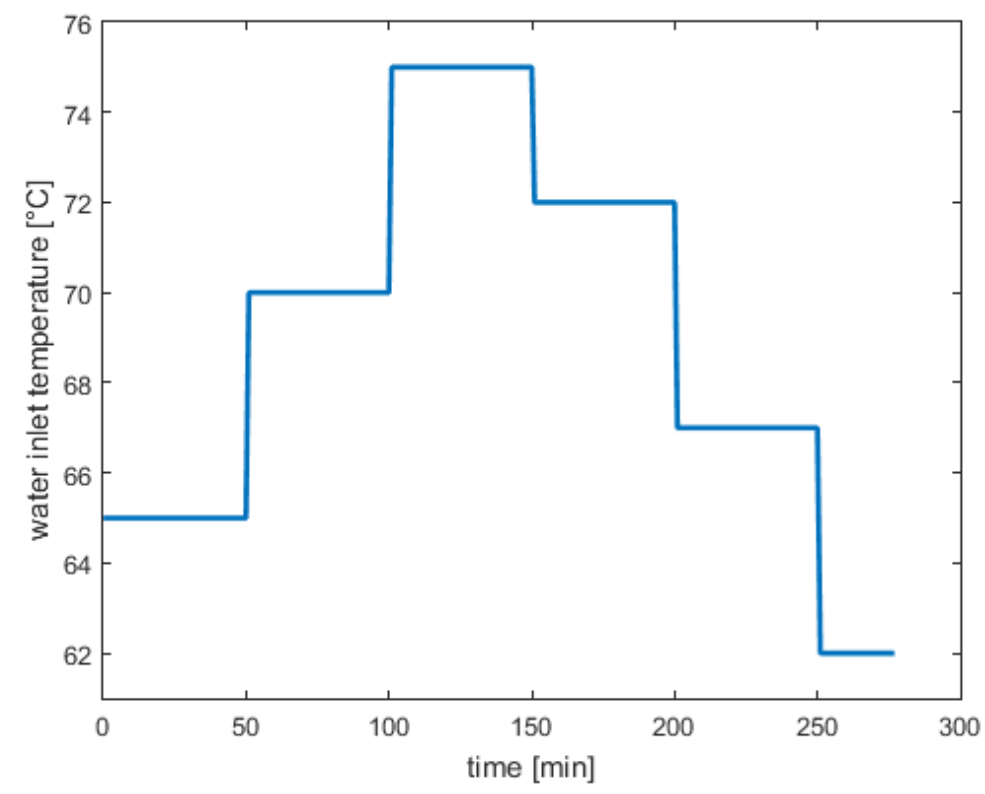

Fig. 2. Inlet water temperature

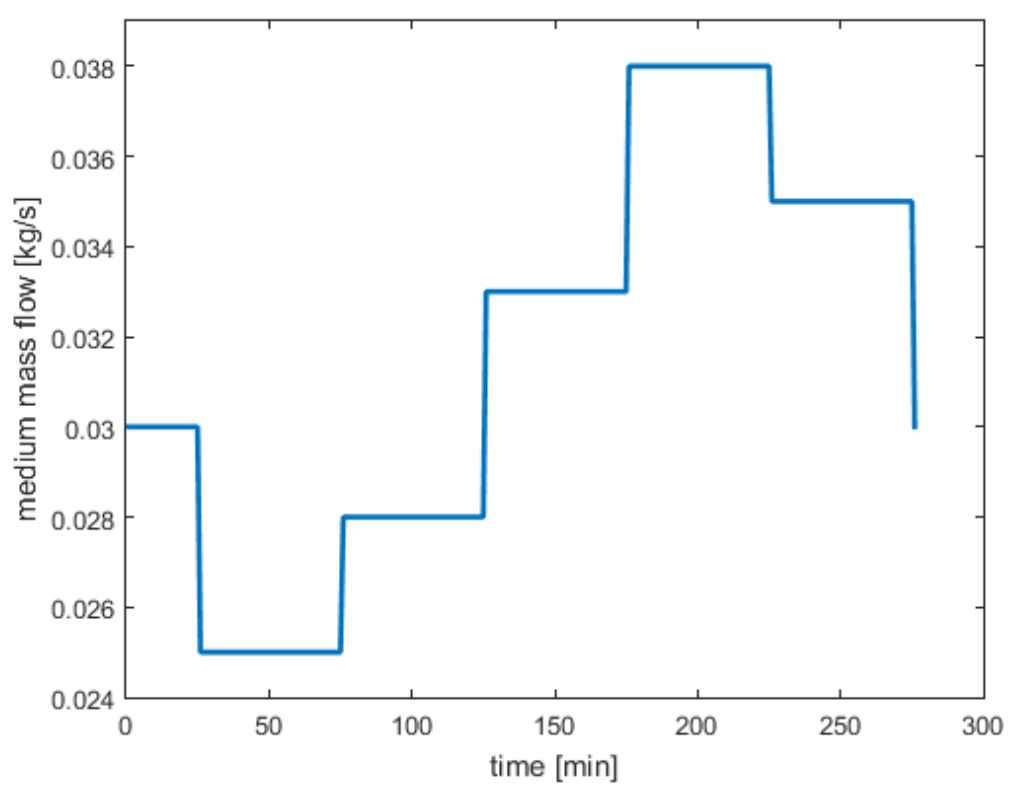

Fig. 3. Medium mass flow

The medium mass flow and inlet water temperature never changed their values at the same time so that their effect on outgoing water temperature could have been seen clearly. The change in either one of these two variables occurred every 25 minutes during the simulation. The inlet water temperature has changed from $62{ }^{\circ} \mathrm{C}$ to $75{ }^{\circ} \mathrm{C}$ and medium mass flow has changed from $0.025 \mathrm{~kg} / \mathrm{s}$ to $0.038 \mathrm{~kg} / \mathrm{s}$.

Continued state-space model of one zone in Faculty of electrical engineering and computing in Zagreb [11] has been used to get the zone temperature $T_{z}$ (Fig. 6). Inputs in that steady-state model were outside temperature and power transmitted between the radiator and the air in the zone, while output was temperature in that zone which was then used as an input in radiator model. Outputs from the radiator model (Fig. 4. and 5.) were the temperature of the radiator outgoing water and radiator skin surface temperature. 


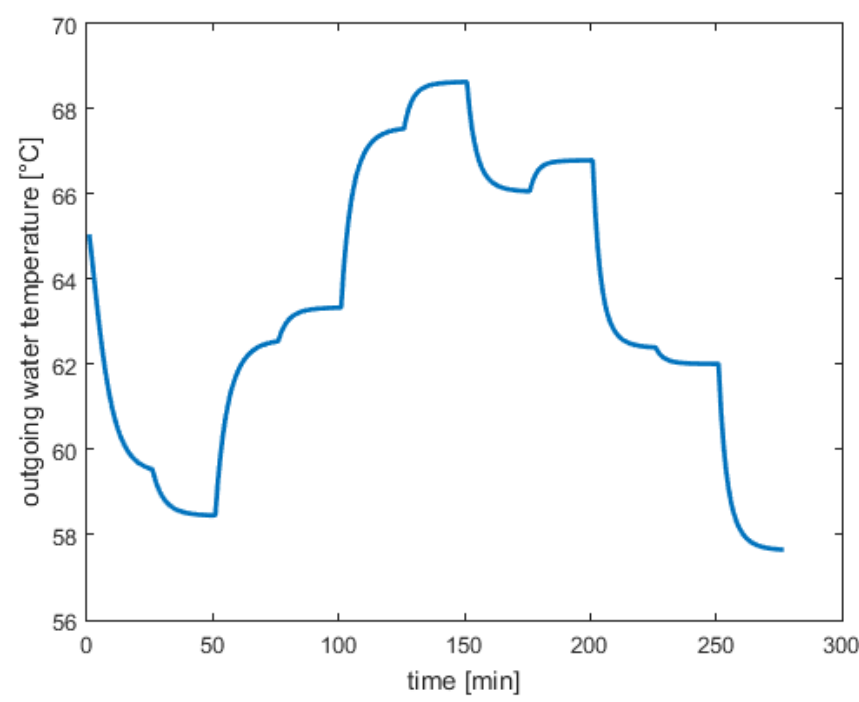

Fig Error! No text of specified style in document.. Temperature of the radiator outgoing water

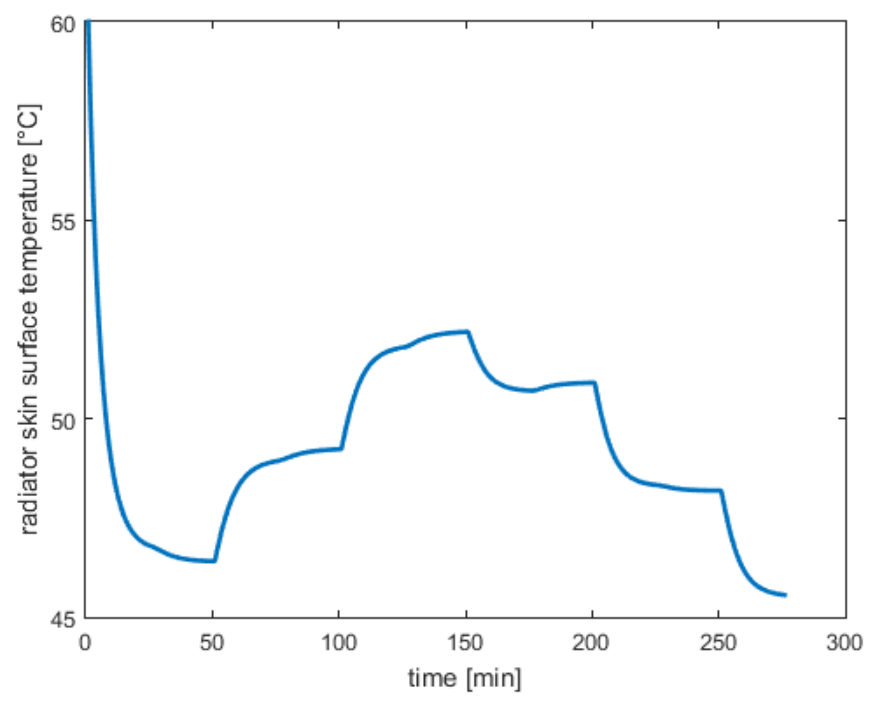

Fig. 5. Temperature of the radiator skin surface

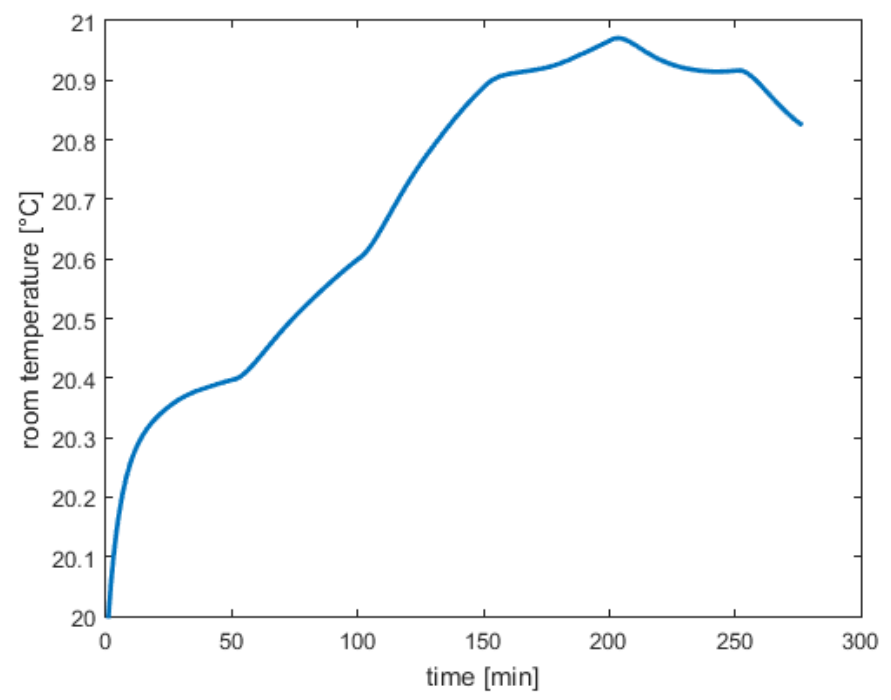

Fig. 6. Zone temperature 


\section{System identification}

The nonlinear optimization procedure has been carried out in MATLAB in order to estimate the unknown parameters $a, b$ and $n$. After that the parameter $U_{0}$ has been identified by using the steady-state approach. In chapter 2 an assumption has been made that the water temperature inside the radiator is the arithmetic average of water inlet temperature and water outlet temperature and that value has also been used in detailed mathematical model of a radiator in Simulink, but with real measurements on radiators in pilot buildings that temperature will maybe significantly differ from arithmetic average of $T_{\mathrm{w}}^{\text {in }}$ and $T_{\mathrm{w}}^{\text {out }}$. To avoid errors, another parameter $C$ is added to identification procedure and it is used to find out how much does each one of these two water temperatures affect the water temperature inside the radiator. The equation (4-4) is now written in the following form:

$\frac{d T_{w, o}}{d t}=a Q_{w}\left(T_{w, i}-T_{w, o}\right)-b\left(C T_{w, i}+(1-C) T_{w, o}-T_{a}\right)^{n}$,

Since we used the arithmetic average of water inlet temperature and water outlet temperature in Simulink model, there is no need for parameter $C$ to be used now because we know that its value is 0.5 , but it will be used on real radiator measurement to eventually improve the identification. The equation (10) is used to calculate the minimized squared error between the measured temperature of outgoing water from the Simulink simulation and simulated temperature of the outgoing water in MATLAB environment.

$\min _{a, b, n, C}\left\|T_{\text {measured }}-T_{\text {sim }}\right\|^{2}$.

By the process of identification the values of unknown parameters have been obtained and they are shown in the Table 1 .

\begin{tabular}{l|llll} 
Model parameters & $A$ & $b$ & $n$ & $C$ \\
\hline Estimated values & $2.259 \cdot 10^{-4}$ & 0.105 & 1.163 & 0.500 \\
\hline
\end{tabular}

Table 1. Estimated radiator parameters

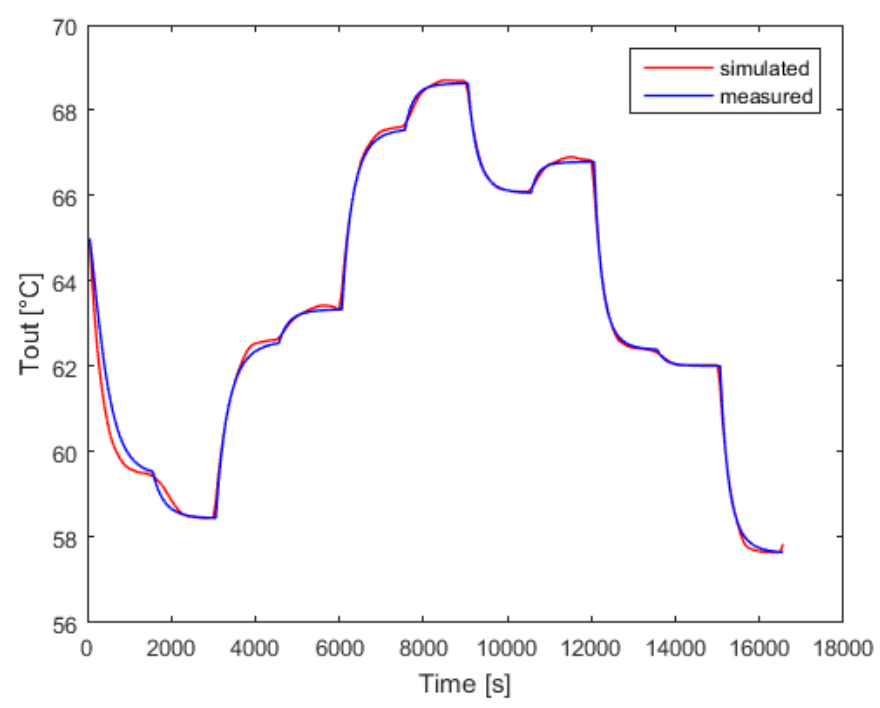

Fig. 7. Measured and identified outgoing water temperature

After the parameters $a, b, n$ and $C$ have been found, there is still unknown value of parameter $U_{0}$ that needs to be estimated, since it has been hidden in the parameter $b\left(b=\frac{U_{0}}{m_{w} c_{w}}\right)$ and thus it can not be estimated directly. The steadystate approach is used to find out $U_{0}$. In steady state there is no change in $T_{w, o}$ and the equation (4-2) is simplified:

$0=q_{w} c_{w}\left(T_{\mathrm{w}}^{\text {in }}-T_{\mathrm{w}}^{\text {out }}\right)-U_{0}\left(T_{\mathrm{w}}^{\mathrm{av}}-T_{z}\right)^{n}$,

In this case, power on the water side $P_{w}$ is equal to the power on the air side $P$ :

$0=q_{w} c_{w}\left(T_{\mathrm{w}}^{\text {in }}-T_{\mathrm{w}}^{\text {out }}\right)-U_{0}\left(T_{\mathrm{w}}^{\mathrm{av}}-T_{z}\right)^{n}$, 
The heat transfer coefficient $U_{0}$ can now be written as:

$U_{0}=\frac{q_{w} c_{w}\left(T_{\mathrm{w}}^{\mathrm{in}}-T_{\mathrm{w}}^{\mathrm{out}}\right)}{\left(T_{\mathrm{w}}^{\mathrm{av}}-T_{z}\right)^{n}}$

Since all the other values in equation are known and $n$ is already estimated, $U_{0}$ can now be identified. 10 steady state values of water-side power $P_{w}$ have been taken and divided by $\left(T_{\mathrm{w}}^{\mathrm{av}}-T_{z}\right)^{n}$ to get 10 values of $U_{0}$ and then the average value is taken as $U_{0}$ (Table 2.). Air-side power $P$ is now known and it is compared to air-side power measured in Simulink. The comparison is shown on the Fig 8 . The biggest difference between these two variables is 14 Watts.

\begin{tabular}{c|c} 
Model parameter & $U_{0}$ \\
\hline Estimated value & 9.17 \\
\hline
\end{tabular}

Table 2. Estimated radiator parameter $U_{0}$

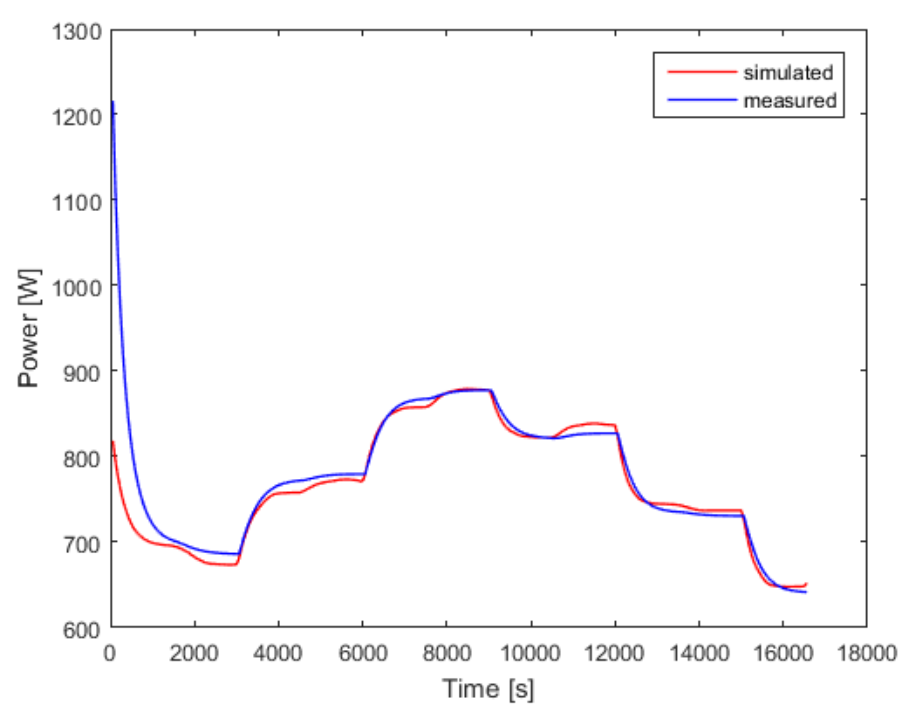

Fig. 8. Measured and identified air-side power

When unknown parameters are identified, the thermal energy aquired by zone actuators can be calculated using the right hand side part of equation (6):

$\int_{0}^{T d} U_{0}\left(T_{\mathrm{w}}^{\mathrm{av}}-T_{z}\right)^{n} d t$.

This equation becomes simpler for the situation with no medium mass flow $\left(q_{w}=0\right)$ :

$\int_{0}^{T d} U_{0}\left(T_{\mathrm{w}}^{\mathrm{out}}-T_{z}\right)^{n} d t$

\section{Conclusion}

This paper presents a mathematical approach to heat transfer processes in hydronic radiator with described identification procedure as a first step towards model predictive control. The outputs from this procedure are the values of unknown parameters needed for calculation of maximum energy that will be used for the MPC. Identification procedure was carried out in Matlab. A zone heated with hydronic radiator was made and simulated in Matlab/Simulink. The results have shown that the identified mathematical model follows the behaviour of the Simulink model very good. The next step is validation of this procedure on real data and the development of control algorithm that will use the described radiator model. The measurement procedure has been described for the purpose of 3Smart project and measuring devices have been installed on the pilot buildings (calorimeters and temperature sensors). The identification procedure described in this paper will be used on all radiators in each pilot building since it is easily replicable. 
This approach enables even larger energy savings by connecting heating and cooling subsystems on zone level (radiators, floor heating, fan coil units) with central HVAC system and that is the main idea of the 3Smart project. However, this procedure has some limitations. It is not optimal to install expensive calorimeters on each radiator in the building, so there will only be one calorimeter per each supplying duct of the building. That means that the overall medium mass flow has to be calculated for each radiator additionally. Also, the heat losses through the pipes will have to be taken into account for the identification on real radiators.

\section{References}

[1] X. Cao, X. Dai, and J. Liu (2016). "Building energy-consumption status worldwide and the state-of-the-art technologies for zero-energy buildings during the past decade," Energy Build., vol. 128, pp. 198-213

[2] I. Hazyuk, C. Ghiaus, and D. Penhouet (2012). "Optimal temperature control of intermittently heated buildings using Model Predictive Control: Part I - Building modeling,” Build. Environ., vol. 51, pp. 379-387

[3] 3SMART project documentation (2017); [Online]. Available: http://www.interreg-danube.eu/approvedprojects/3smart

[4] B. Crnokic, G. Ljesic, Z. Stojkic, and M. Bandic Glavas (2017). "Analysis of Energy Consumption in the Building as the Basis for Development of the Modular Model Predictive Control System," Proceedings of the 28th DAAAM International Symposium, pp. 0299-0308, B. Katalinic (Ed.), Published by DAAAM International, ISBN 978-3-902734-xx-x, ISSN 1726-9679, Vienna, Austria

[5] M. Embaye, R. K. AL-Dadah, and S. Mahmoud (2014). "Effect of flow pulsation on energy consumption of a radiator in a centrally heated building," Int. J. Low-Carbon Technol., vol. 11, no. 1, p. ctu024

[6] C. Brembilla, M. Soleimani-Mohseni, and T. Olofsson (2015). "Transient model of a panel radiator," in Proceedings of BS2015: 14th Conference of International Building Performance Simulation Association, Hyderabad, India

[7] P.-O. Johansson and J. Wollerstrand (2010). "Heat Output from Space Heating Radiator with Add-on-fan on fan Blowers," COMSOL Conf.

[8] I. Martínez (1992). Termodinámica básica y aplicada. Dossat

[9] MATLAB (2015). version 8.5.0 (R2015a). The MathWorks Inc.

[10] Simulink - Simulation and Model-Based Design - MATLAB \&amp; Simulink (2017); [Online]. Available: https://www.mathworks.com/products/simulink.html

[11] A. Martincevic, A. Starcic, and M. Vasak (2014). "Parameter estimation for low-order models of complex buildings," in IEEE PES Innovative Smart Grid Technologies, Europe, pp. 1-6 